\title{
IRX1 Promotes Loss of Subcutaneous Adipose Tissue by Inhibiting Adipocyte Differentiation in Cachectic Patients with Gastric Cancer
}

Jun Han

Zhongshan Hospital

Zuoyou Ding

Zhongshan Hospital

Qiulin Zhuang

Zhongshan Hospital

Lei Shen

Zhongshan Hospital

Fan Yang

Zhongshan Hospital

Szechun Sah

Zhongshan Hospital

Guohao Wu ( $\nabla$ profwugh@163.com )

Zhongshan Hospital

\section{Research Article}

Keywords: Cancer cachexia, Adipose tissue loss, Adipogenesis, Subcutaneous adipose tissue, IRX1

Posted Date: February 16th, 2022

DOI: https://doi.org/10.21203/rs.3.rs-1344846/v1

License: (c) (1) This work is licensed under a Creative Commons Attribution 4.0 International License. Read Full License 


\section{Abstract}

Background: Loss of adipose tissue is one of the most important characteristics of cancer cachexia. The loss degree of subcutaneous adipose tissue (SAT) and visceral adipose tissue (VAT) is different in the same cachectic patient. However, it is unclear which genes lead to the differential fat loss in cancer cachexia. The purpose of this study is to screen the differentially expressed genes (DEGs) profile of SAT and VAT and clarify its function in cancer cachexia.

Methods: We screened the DEGs of SAT and VAT in cachectic patients with gastric cancer by RNA sequencing. Gene enrichment analyses were preformed to analyze the biological function of DEGs. Interaction network was constructed to illustrate the relationship among key genes. Clinical significance of IRX1 related to cancer cachexia was illustrated by Spearman's correlation analysis. SAT and VAT samples were collected and performed histomorphology analyses. Mouse model of cancer cachexia was established and differences between SAT and VAT were compared. The function of IRX1 on lipid metabolism was clarified by Oil Red $\mathrm{O}$ staining, QPCR, and western blotting in adipocytes.

Results: A total of 455 DEGs were screened between SAT and VAT in cachectic patients with gastric cancer. Gene enrichment analysis indicated that the immune response might contribute to the pathological difference of SAT and VAT in caner cachexia. Patients with higher expression of IRX1 in SAT than VAT revealed significantly higher weight loss, IL-6 and TNFa concentration, as well as lower BMI, apoE, SAT and VAT area. A greater loss in SAT was observed in cachectic mice comparing to noncachectic mice while the expression of IRX1 in SAT of cachectic mice was significantly higher. Oil Red 0 staining showed that IRX1 inhibited lipogenesis in adipocytes. Significant changes in the genes related to adipocyte differentiation and adipogenesis were observed in both the cases of IRX1 overexpression and knockdown.

Conclusion: Higher expression of IRX1 was observed in SAT than VAT in gastric cancer patients with cachexia and thus IRX1 promotes loss of SAT by inhibiting adipocyte differentiation and adipogenesis. IRX1 is expected to be a potential target for the prevention and treatment of SAT loss in cancer cachexia.

\section{Introduction}

Cancer cachexia is a clinical syndrome characterized by muscle atrophy and fat loss[1]. As an important feature of patients with cancer cachexia, fat loss can reduce chemotherapy tolerance and is an independent prognostic factor for poor prognosis[2]. Our previous studies have also shown that fat loss is related to poor nutritional status and high inflammatory factors in patients with cancer cachexia[3, 4]. It is also reported that fat loss occurs earlier than muscle atrophy in cachexic patients[5]. Therefore, the role and mechanism of fat loss in cancer cachexia have attracted much attention.

The anatomical location of the fat depot influences adipose tissue metabolism and function. SAT comprises more than $80 \%$ of the total body fat, whereas VAT comprises up to $10 \%$ and $20 \%$ of the total body fat, respectively[6]. CT image analysis enables precise quantification of adipose depots and has 
emerged as the golden standard to segment SAT and VAT[7].Not only do SAT and VAT differ in anatomic location, but also in endocrine function, adipokine secretion and lipolytic activity [8,9]. For example, the accumulation of VATcontributes to obesity related diseases, such as metabolic syndrome[10]. Accumulation of VAT is also a high-risk factor for colorectal cancer and other tumors[11, 12]. On the contrary, SAT accumulation, if not brings a protective effect, is unlikely todo harminmetabolism of patients.These metabolic differences demonstrate the necessity to evaluate the effect based on SAT and VAT respectively rather than total adipose tissue. Our previous research has already found that the loss of SAT was greater than the loss of VAT in a cohort study of 411 gastric cancer patients with cachexia[13].And the SAT area index was an independent risk factor for poor prognosis in patients with gastric cancer cachexia. Hence, it is rather essential to evaluate the different gene profile between SAT and VAT, and clarify the key genes that prompt the greater loss of SAT than VAT. Although genes were reported to be differentially expressed in SAT and VAT in obese patients[14],theDEGsbetween SAT and VAT in gastric cancer patients with cachexia has not been fully explored before.

It has beenconfirmed that the main mechanisms of adipose tissue lossin cancer cachexia include activation of lipolysis, white-to-brown trans-differentiation of WAT (WAT browning), and dysfunctional adipogenesis[15]. Our previous research has proved these points from multiple perspectives. Interleukin-6 (IL-6)was proved to induce adipose tissue loss in patients with cancer cachexia by promoting lipolysis and WAT browning[4]. LncRNA-CAAlnc1 couldsuppress adipogenesis by blocking the binding of HuR in adipose tissue of cancer cachexia patients[16]. MiR-410-3p could down-regulate IRS-1 and PPAR-y to inhibitadipogenesis and lipid accumulation[17]. CircPTK2 was upregulated in adipose tissues of cachectic patients and induced adipose tissue loss by promoting lipolysis and inhibiting adipogenesis[18]. However, these studies have mainly focused on the DEGs of SAT between cachectic patients and non-cachectic patients. The DEGs betweenSAT and VAT in cachectic patients and the key genes among them remain unclear.

In this study, RNA sequencing was performed to screen DEGsbetween SAT and VAT of gastric cancer patients with cachexia. Clinicopathological significance of DEGs was analyzed in a large number of samples of gastric cancer patients with cachexia. In addition, DEGs were further verified in cancer cachexia mouse models. The function of the DEGswas analyzedby gain and loss of function in preadipocytes.

\section{Material And Methods}

\section{Patients}

Cachectic patients with gastric cancerwho underwentsurgery in Zhongshan Hospital of Fudan University from 2018.06 to 2020.06 were includedin this study. Inclusive criteria for the study were: (1) patients diagnosed with gastric adenocarcinomacancer (not gastric stromal tumor orlymphoma); (2) patients received surgical treatmentwithout preoperative radiotherapy or chemotherapy; (3) patients with abdominal CT examinations and complete clinical data; (4) patients with weight loss $>5 \%$ in recent 6 
months before surgery. This study was approved by the Ethics Committee of Zhongshan Hospital of Fudan University (B2019-193R). Written informed consents were obtained from all patients.

\section{Clinical data collection}

Height, weight, gender, and age were extracted from the preoperative medical records. Body mass index (BMI) was calculated as body weight $(\mathrm{kg}) /$ height $^{2}\left(\mathrm{~m}^{2}\right)$.Cachexia-related indicators were extracted from preoperative blood biochemical examinations. Areas of SAT and VAT from CT scans at the third lumbar vertebra were measuredas described before[13].

\section{Human tissue specimens}

Adipose tissues were obtained from enrolled patients. At the beginning of the operation, about $500 \mathrm{mg}$ of subcutaneous adipose tissue near the median abdominal incision was obtained. About $500 \mathrm{mg}$ of omental adipose tissue was taken as visceral adipose tissue within 30 minutes after gastric cancer specimens were isolated. The adipose tissue was immediately stored in liquid nitrogen at $-80{ }^{\circ} \mathrm{C}$ or transferred into tissue fixative for further analysis.

\section{Mouse model of cancer cachexia}

The mouse model of cancer cachexia refers to the previous methods[4]. In brief, Cachectic mice were induced by subcutaneous injection of colon- 26 adenocarcinoma cells into the right flank of the mice. The littermate control mice received PBS injection only. Micewere euthanized at day 21 post-injection and were dissected toharvestinguinal white adipose tissue (SAT) and epididymal white adipose tissue (VAT).

Weight of SAT and VAT was recorded at day 0 (control mice) and day 21 (cachectic mice). The proportion of adipose tissues loss was calculated by weight change (weight at day 0subtract weight at day 21) divided to initial weight. Allanimal studies were performed in accordance with the guidelinesprovided by Animal Care Committee of Fudan University.

\section{RNA sequencing}

The total RNA was extracted from 3 paired SAT and VAT from gastric cancer patients with cachexia. After quantification and qualification, a total amount of $1 \mu \mathrm{g}$ RNA per sample was used as input material for the RNAsample preparations. Sequencing libraries were generated using NEBNextUltraTM RNA Library Prep Kit for Illumina (NEB, USA) following manufacturer's recommendations and index codes were added to attribute sequences to each sample. The clustering of the index-coded samples was performed on a cBot Cluster Generation System using TruSeq PE Cluster Kit v3-cBot-HS (Illumina, USA) according to the manufacturer's instructions. After cluster generation, the library preparations were sequenced on an Illumina Novaseq platform and 150bp paired-end reads were generated. FeatureCounts v1.5.0-p3 was used to count the reads numbers mapped to each gene. Fragments per kilobase million (FPKM) of each gene was calculated based on the length of the gene and reads count mapped to this gene. The raw 
sequencing dataset that supported the resultsof this study was deposited in the NCBI GEO database. The data areaccessible through GEO: GSE186466.

\section{RNA sequencing data analysis}

Differential expression analysis of twogroupswas performed using theDESeq2 R package. Differentially expressed transcripts between the two groups were identified when |logFoldChange| $>0$ and the $p$ value $<0.05$. Gene Ontology (GO) enrichment analysis of DEGs wasimplemented by the ClusterProfiler $R$ package, in which gene length biaswascorrected. We also used ClusterProfiler $\mathrm{R}$ package to test thestatistical enrichment of differential expression genes in KEGG pathways. GO terms and KEGG pathways with corrected $p$ value $<0.05$ were consideredsignificantly enriched by differential expressed genes.

Conventional enrichment analysis based on hypergeometric distribution depends on significantly upregulated or down-regulated genes, and it is easy to omit some genes with insignificant differential expression but important biological significance. Gene set enrichment analysis (GSEA) does not need to specify a clear differential gene threshold. All genes are sorted according to the degree of differential expression in the two groups of samples, and then statistical methods are used to test whether the preset gene set is enriched at the top or low section of the sorting table. GSEA mainly includes three steps: calculation of enrichment score; estimation of the significance level of enrichment score; multiple hypothesis tests.

The PPI network of DEGs was predicted using the Search Tool for the Retrieval of Interacting Genes (STRING) database. The interaction score threshold of 0.4 was set as the cut-off criterion. The PPI network was constructed using Cytoscape. Comprehensive experimentally validated miRNA-gene interaction data were collected from TargetBase. Transcription factor and gene target data derived from the ENCODE ChIP-seq data. Only peak intensity signal $<500$ and the predicted regulatory potential score $<1$ is used (using BETA Minus algorithm).

\section{Cell culture and differentiation}

The mouse immortalized white preadipocytes werekindly provided by Professor Qiurong Ding from the Shanghai Institute of Nutrition and Health, Chinese Academy of Sciences. Thiscell line has been described previously and used in several studies toassess the effects of different factors on adipose differentiation andfunction[19]. The culture and differentiation methods of preadipocytes cell lines were as previously reported[18].

\section{Plasmid and siRNA construction}

The IRX1 expression plasmid pcDNA3.1p/IRX1 and empty plasmid pcDNA3.1p were designed and synthesized by GenePharma (Shanghai, China). Small interfering RNAs (siRNAs) targeting IRX1 were also designed by GenePharma. Cell transfection was conducted using Lipofectamine RNAiMAX Transfection 
Reagent kit (Invitrogen,USA) or Lipofectamine 2000 Transfection Reagent (Invitrogen, USA) according to the manufacturer's instruction

\section{Hematoxylin-eosin and Immunohistochemical staining}

All the samples were transferred totissue fixativeafter being harvested. The protocols were previously described[18]. In brief, histological sections of adipose tissue were stained with hematoxylin-eosin to evaluate morphological changes and the adipocyte cross-sectional area (CSA). A total of 10 randomly selected fields for each sectionwere captured and analyzed to evaluate adipocyte CSA with a computerized imaging software (ImageJ, USA).For immunohistochemistry, the positive cellsin 10 randomly selected fields per section were counted and evaluated by two independent researchers. And the mean number of positive cells per field was calculated.

\section{Oil Red 0 (ORO) staining}

Mature adipocytes were fixed with $4 \%$ formaldehyde for $30 \mathrm{~min}$, then washed twice with PBS. They were stained with $0.3 \%$ ORO solution and washed three times with distilled water. To assess lipid accumulation, the dye retained in the cells was dissolved in isopropanol and the absorbance of the resulting solution at $520 \mathrm{~nm}$ was examined.

\section{RNA isolation and qRT-PCR}

Total RNA was isolated from adipose tissues and adipocytes using TRIzol Reagent (Invitrogen, USA) according to the manufacturer's recommendations.cDNA was synthesized from $1 \mu \mathrm{g}$ total RNA using FastKing RT Kit (Tiangen, China).Gene expression analysis was performed using Prime-Script RT master mix (Takara, Japan) in StepOnePlusReal-Time system (Applied Biosystems, USA). Expression levels of targeted geneswerenormalized to the expression of GAPDH. qRT-PCR was performedaccording to the manufacturer's instructions and the relativefold change was calculated by the $2^{-\Delta \Delta C t}$ method. Primers were designed and synthesized by Sangon Biotech (Shanghai, China) and are listed in Additional file: Table S2. All experiments were repeated at least three times.

\section{Western blot analysis}

Preparation of total protein lysates and western blot analysis were performed as previously described[16]. Primary antibodiesagainst IRX1 (Immunoway,YT2412), CEBPa (Cell signaling technology, \#2295),AdipoQ(Cell signaling technology, \#2789), and FABP4(Cell signaling technology, \#2120)were used. Tubulin expression wasused as an endogenous control.

\section{Statistical analyses}

Statistical analyses were performed using GraphPad Prism software. Data calculated from independent experiments were presented as the mean \pm standard deviation and a student's $t$-test was performed to compare the differences between two groups. To analyze the correlation between IRX1 mRNA levels in 
SAT and the clinicopathological factors in gastric cancer patients with cachexia, we divided 61 patients into two groups according to the IRX1expression in SATcompared to VAT. Comparisons between these two groups were made using thet-test for continuous data and $\chi^{2}$ test for categorical data. $p<0.05$ was considered statistically significant.

\section{Results}

\section{Identification of DEGs between SAT and VAT}

To investigate DEGs between SAT and VAT in patients with cachexia, we performed whole transcriptome RNA sequencing of SAT and VAT from 3 patients. Their clinical features and typical CT image at the third lumbar vertebra were shown in Table S1 and Figure S1.Gene expression distributions of each sample were shown in Figure S2.As illustrated by volcano plot in Figure 1A, a total of 455 DEGs were detected in SAT compared to VAT ( $p<0.05$ and $|\log \mathrm{FC}|>0)$ ), including 342 downregulated mRNAs and 113 upregulated mRNAs. Cluster analysis of DEGs was shown in Figure 1B by heatmap. Thetop 10downregulated DEGs and top 10 upregulated DEGs were shown in Table 1.To better understand the functional implications of 455 DEGs betweenSAT and VAT in cachectic patients, we performed GO analysis and KEGG analysis, and GSEA analysis. The GO terms were shown in Figure 1C. We found that the leukocyte migration, humoral immune response, complement activation, adaptive immune response , and humoral immune response mediated by circulating immunoglobulin were among the top $10 \mathrm{GO}$ terms, which indicated that the immune response might play an essential role in different functions and mechanisms between SAT and VAT.Interestingly, cytokine-cytokine receptor interaction, complement and coagulation cascades, and chemokine signaling pathway weretop 3 of the KEGG pathways(Figure 1D), which also indicated thesignificance of immune response between SAT and VAT.

\section{GSEA analysis and interaction network of DEGs}

GSEA analysis was conducted to achieve further insight intothe biologic pathways involved in different adipose tissue distribution of cachectic patients. As shown in Figure 2A-2C, we found that lipid catabolic process, regulation of fatty acid beta-oxidation, and positive regulation of triglyceride metabolic process were enriched in SAT group. On the contrary, we found that negative regulation of chondrocyte differentiation, positive regulation of calcium-mediated signaling, and negative regulation of muscle cell apoptotic process were enriched in VATgroup (Figure 2D-2F). The network of DEGsbetween SAT and VAT wasconstructed by STRING, with a threshold of 0.4. The PPI network with 205 nodes and 720 edges wasdisplayed by Cytoscape-MCODE (Figure 3A). Then we constructed an interaction network linking top 10 DEGs to their relevant miRNAs/translation factors.As shown in Figure 3B-3C,HOXC8, HOXC10, and IRX5 were key genes of the regulating network.

\section{Expression of IRX1 was higher in SAT than VAT in cachectic patients}

Among the most differentially expressed genes, we found that IRX1, which encodes a member of the Iroquois homeobox protein family, is highly expressed in SAT compared to VAT.To further clarify the 
clinical significance of different expression of IRX1 between SAT and VAT, we performed qRT-PCR assay and showed that IRX1 was significantly upregulated in SATcompared with VAT $(p<0.05$, Figure

4A).Among the 61 pairs of adipose tissues, we found that IRX1 was higher expressed in SAT than VAT in 37 pairs and lower expressed in SAT than VAT in 24 pairs. Then, we divided them into two groups and analyzed the clinicopathological correlation between groups. The analysis showed that weight loss, IL6, and TNFa were significantly increased in cachectic patients with higherIRX1 expression in SAT than VAT. While BMI, apoE, SAT area and VAT area were significantly decreased in cachectic patients with higherIRX1 expression in SAT than VAT (Table 2).In addition, correlationanalysis was performed between IRX1 expression and adipose tissue area. The results of the Spearman's correlation analysis showed that the expression level of IRX1 in WAT was negatively correlated with WAT area (Figure 4B), and the expression level of IRX1 in VAT revealed no correlation with VAT area(Figure 4C). To further validate our hypothesis, HE staining and IHC were performed in WAT and VAT from cachectic patients. The representative images of $\mathrm{HE}$ staining revealed that there is no significant difference between adipocytes of SAT and VAT in terms of morphology(Figure 4D). The representative imagesof IHC confirmed that there is a significant increase in IRX1 expression of adipocytes in SAT with respect to VAT (Figure 4E, $p<0.05)$. These results suggested that IRX 1 is involved inthe lipid metabolism ofthe pathological process in cachexia.

\section{Expression of IRX1 was higher in SAT than VAT in mouse model of cachexia}

To further validate our findings in animal models, we construct a mouse modelof cancer cachexiaas described before. As shown in Figure 5A-5B, it became clear that adipocytesmorphologydid not reveal significant difference between different fat depots of the same cachectic mice. Yet the IRX1 expression of adipocytes in SAT is significantly higher than that in VAT (Figure 5C-5D). To calculate the loss ratio of different fat depots, we recordweight of SAT and VAT at day 0 (control mice) and day 21 (cachectic mice). The result showed that loss ratio of SAT was significantly higher than that of VAT in cachectic mice (Figure 5E). Then we compared the mRNA and protein levels of IRX1 in SAT and VAT of cancercachexia mice. The results showed that the mRNA and protein expression levels of IRX1 inSAT were higher than that in VAT in cancer cachexia mice (Figure 5F-5G), which was consistent with the resultsabove.

\section{IRX1 inhibited adipogenesis in adipocytes}

To investigate the role of IRX1 in adipocyte metabolism, IRX1 was upregulated by transfecting preadipocyte with IRX1 overexpression vectors, followed by induction and differentiation. The qPCRresult showed that IRX1 expression had increased about ten-fold bydifferentiation day 6 in the IRX1 overexpression group compared tothe control group (Figure 6A). We detected the changes of adipogenesis-related genes involved in the lipid metabolismafterlRX1 overexpression. The results of qPCR and western blottingshowed that AdipoQ, FABP4, and CEBPa were significantly down-regulated when IRX1 was overexpressed (Figure 6B-6C). IRX1 overexpression also resulted in decreasing in lipid dropletaccording to the Oil Red 0 staining (Figure 6D). To further demonstrate whether IRX1 was required for adipocyte metabolism, adipocytes weretransfected with three independent siRNAs. Effective 
knockdown was achieved by two of the siRNAs (Figure 6E). The results of qPCR and western blottingshowed that AdipoQ, FABP4, and CEBPawere also significantly up-regulatedwhen IRX1 was knockdown (Figure 6F-6G). The results of Oil Red 0 stainingwere consistent with the findings above (Figure 6H).

\section{Discussion}

The role of fat loss in patients with cancer cachexia is becoming a heat topic. SAT and VAT play different roles in the development of cancer cachexia. However, there is no systematic study on the difference of gene expression between SAT and VAT in gastric cancer patients with cachexia. In this study, the mRNA expression profiles of SAT and VAT in patients with cancer cachexia were screened by RNA sequencing for the first time.342 downregulated and 113 upregulated mRNAs were screened compared SAT to VAT in patients with gastric cancer cachexia. IRX1 was one of the most highly expressed mRNAs in SATboth in cachexic patients and mice. It is observed that weight loss, IL6, and TNFa were significantly increased while BMI, apoE, SAT area and VAT area were significantly decreased in cachectic patients with higher IRX1 expression in SAT than VAT.In vitro experiments showed that IRX1 promotesfat loss by inhibiting lipid differentiation and adipogenesis.

The metabolic differences between SAT and VAT were mostly studied in obese patients. The adipose tissues in both fat depotsare similar in their morphologyand lipid storage functions. As shown in present research, adipocytes from SAT an VAT in both cachectic patients and mice revealed evident atrophy, yet no significant difference was observed regarding the morphology of adipocytes. Nonetheless, emerging evidence has suggested that SAT and VAT have different characteristicsand functional roles in metabolic regulation[20,21]. Thevisceral depot is often considered more deleterious than thesubcutaneous depot. Two independent research demonstrated that VAT hypertrophy is predominantly associated with dyslipidemia whereas SAT hypertrophy is mainly associated with insulin resistance in humans[22]. However, metabolic differences between SAT and VAT in cancer cachexia have not been reported. In this study, we detected hundreds of DEGs between SAT and VAT in gastriccancer patients with cachexia by mRNA sequencing. GO and KEGG analysis showed that immune response might be involved in the difference between SAT and VAT, which was consistent with the differential expression of immune cells in SAT and VAT by single cell sequencing[23]. However, the functions of specific types of immune cells and their mechanisms during the development of cancer cachexiaremain further clarified.

IRX1, a member of the Iroquois homeobox family of transcription factors, played a crucial role inembryonic development[24]. Recently, IRX1 was identified as apotential tumorsuppressor gene in head and neck squamous cellcarcinoma and gastric cancer[25-27]. However,IRX1 also acted as a metastaticoncogene which was activated by hypomethylation[28]. As far as we know, the role ofIRX1 in the progression of fat loss during cancer cachexia has not been reported. In this study, we found IRX1 was significantly upregulated in SAT compared with VAT in both human and mice. IRX1 expression in SAT was found to benegatively correlated with SAT area andwas involved in the lipid metabolism of the 
pathological process in cachexia. These results indicated IRX1 gene plays an important role in promoting the loss of SAT during cancer cachexia.

We firstly reported that overexpression of IRX1 inhibited adipogenesisin preadipocytes. Activation of lipolysis and WAT browning or dysfunctional adipose differentiation and adipogenesis were reported to be the main mechanism of fat loss in cancer cachexia[29]. By overexpression and knockdown of IRX1, we clarifiedthat IRX1 exerted its function by inhibiting adipogenesis in the lipid metabolism. These results suggested that adipose differentiation and adipogenesis related genes might be important in the regulation of lipid metabolism during cancer cachexia, which was consistent with our previous foundation.In future research, we will further explore the specific molecular mechanism of IRX1 regulating adipose differentiation and adipogenesis related genes.

\section{Conclusion}

Inconclusion, we screened mRNA expression profiles of SAT and VAT in patients with cancer cachexia by RNA sequencing. Hundreds of DEGs were detected inSAT and VAT and IRX1 wasone of thehighly expressed mRNAs in SAT. Clinical studies showed thatIRX1 expression in SAT was negatively correlated with SAT area.In vitro experiments showed that IRX1 inhibited lipogenesis by modulating differentiation and adipogenesis related genes. Our results demonstrated that IRX1 was expected to be a potential target for the prevention and treatment of SAT loss in cancer cachexia.

\section{Abbreviations}

WAT: White adipose tissue; SAT: Subcutaneous adipose tissue; VAT: Visceral adipose tissue; FPKM: Fragments per kilobase million; GO: Gene Ontology; KEGG: Kyoto Encyclopedia and Genes and Genomes; GSEA: Gene set enrichment analysis; ORO: Oil Red O; BMI: Body mass index; IncRNA: Long non-coding RNA; circRNA: Circular RNA; siRNA: Small interfering RNAs; ceRNA: Competing endogenouse RNA; IL-6: Interleukin- 6; TNF-a: Tumor necrosis factor-a; FABP4: Fatty acid binding protein 4; CEBPa: CCAAT/enhancer binding proteins a; IRX1: Iroquois homeobox 1.

\section{Declarations}

\section{Acknowledgements}

We thank Prof. Qiurong Ding from Shanghai Institute of Nutrition and Health, Shanghai Institutes for Biological Sciences, Chinese Academy of Sciences for kindly providing the immortalized subcutaneous white preadipocytes.

\section{Authors' Contribution}

J.H. and G.W. conceived, designed, and drafted the manuscript. Q.Z., L.S., F.Y. and S.S. collected the clinical data. J.H and Z.D performed the experiments. J.H., Z.D. and Q.Z. conducted the statistical 
analyses. J.H. drafted the manuscript. G.W. and J.H. revised the manuscript. All authors read and approved the final manuscript.

\section{Funding}

This work was supported by the Youth of National Natural Science Foundation of China (81803091).

\section{Availability of Data and Materials}

RNA sequencing data were deposited at the GEO database with the accession number GSE1186466.All data generated or analyzed during this study are included in this published article and its supplementary information files.

\section{Ethics Approval and Consent to Participate}

This study was conducted according to the standards of the Declaration of Helsinki. Approval by the Ethics Committee of Zhongshan Hospital, Fudan University was obtained. Written informed consents were obtained from all participants. All procedures described involving animals were approved by the Animal Care Committee of Fudan University and conducted in accordance with the guidelines of Fudan University on the experimental use of laboratory animals.

\section{Competing Interests}

The authors declare that they have no competing interests.

\section{Consent for Publication}

Not applicable.

\section{References}

1. Fearon K, Strasser F, Anker SD, Bosaeus I, Bruera E, Fainsinger RL, et al: Definition and classification of cancer cachexia: an international consensus.Lancet Oncol 2011, 12:489-495.

2. Baracos VE, Martin L, Korc M, Guttridge DC, Fearon KCH: Cancer-associated cachexia.Nat Rev Dis Primers 2018, 4:17105.

3. Han J, Lu C, Meng Q, Halim A, Yean TJ, Wu G: Plasma concentration of interleukin-6 was upregulated in cancer cachexia patients and was positively correlated with plasma free fatty acid in female patients.Nutr Metab (Lond) 2019, 16:80.

4. Han J, Meng Q, Shen L, Wu G: Interleukin-6 induces fat loss in cancer cachexia by promoting white adipose tissue lipolysis and browning.Lipids Health Dis 2018, 17:14.

5. Tsoli M, Swarbrick MM, Robertson GR: Lipolytic and thermogenic depletion of adipose tissue in cancer cachexia.Semin Cell Dev Biol 2016, 54:68-81. 
6. Morigny P, Boucher J, Arner P, Langin D: Lipid and glucose metabolism in white adipocytes: pathways, dysfunction and therapeutics.Nat Rev Endocrinol 2021, 17:276-295.

7. Kim YJ, Lee SH, Kim TY, Park JY, Choi SH, Kim KG: Body fat assessment method using CT images with separation mask algorithm.J Digit Imaging 2013, 26:155-162.

8. Chau YY, Bandiera R, Serrels A, Martínez-Estrada OM, Qing W, Lee M, et al: Visceral and subcutaneous fat have different origins and evidence supports a mesothelial source.Nat Cell Biol 2014, 16:367-375.

9. Porro S, Genchi VA, Cignarelli A, Natalicchio A, Laviola L, Giorgino F, et al: Dysmetabolic adipose tissue in obesity: morphological and functional characteristics of adipose stem cells and mature adipocytes in healthy and unhealthy obese subjects.J Endocrinol Invest 2021, 44:921-941.

10. Zhen Y, Shu W, Hou X, Wang Y: Innate Immune System Orchestrates Metabolic Homeostasis and Dysfunction in Visceral Adipose Tissue During Obesity.Front Immuno/ 2021, 12:702835.

11. Keum N, Lee DH, Kim R, Greenwood DC, Giovannucci EL: Visceral adiposity and colorectal adenomas: dose-response meta-analysis of observational studies.Ann Oncol2015, 26:1101-1109.

12. Iwase $T$, Wang $X$, Shrimanker TV, Kolonin MG, Ueno NT: Body composition and breast cancer risk and treatment: mechanisms and impact.Breast Cancer Res Treat 2021, 186:273-283.

13. Han J, Tang M, Lu C, Shen L, She J, Wu G: Subcutaneous, but not visceral, adipose tissue as a marker for prognosis in gastric cancer patients with cachexia.Clin Nutr 2021, 40:5156-5161.

14. Hwang I, Kim JB: Two Faces of White Adipose Tissue with Heterogeneous Adipogenic Progenitors.Diabetes Metab J 2019, 43:752-762.

15. Sun X, Feng X, Wu X, Lu Y, Chen K, Ye Y: Fat Wasting Is Damaging: Role of Adipose Tissue in CancerAssociated Cachexia.Front Cell Dev Biol 2020, 8:33.

16. Shen L, Han J, Wang H, Meng Q, Chen L, Liu Y, et al: Cachexia-related long noncoding RNA, CAAlnc1, suppresses adipogenesis by blocking the binding of HuR to adipogenic transcription factor mRNAs.Int J Cancer 2019, 145:1809-1821.

17. Sun D, Ding Z, Shen L, Yang F, Han J, Wu G: miR-410-3P inhibits adipocyte differentiation by targeting IRS-1 in cancer-associated cachexia patients.Lipids Health Dis 2021, 20:115.

18. Ding Z, Sun D, Han J, Shen L, Yang F, Sah S, et al: Novel noncoding RNA CircPTK2 regulates lipolysis and adipogenesis in cachexia.Mol Metab 2021, 53:101310.

19. Qiu Y, Sun Y, Xu D, Yang Y, Liu X, Wei Y, et al: Screening of FDA-approved drugs identifies sutent as a modulator of UCP1 expression in brown adipose tissue.EBioMedicine 2018, 37:344-355.

20. Ibrahim MM: Subcutaneous and visceral adipose tissue: structural and functional differences.Obes Rev 2010, 11:11-18.

21. Abe T, Song JS, Bell ZW, Wong V, Spitz RW, Yamada Y, et al: Comparisons of calorie restriction and structured exercise on reductions in visceral and abdominal subcutaneous adipose tissue: a systematic review.Eur J Clin Nutr 2021. 
22. Wijetunge S, Ratnayake R, Kotakadeniya H, Rosairo S, Albracht-Schulte $K$, Ramalingam L, et al: Association between serum and adipose tissue resistin with dysglycemia in South Asian women.Nutr Diabetes 2019, 9:5.

23. Vijay J, Gauthier MF, Biswell RL, Louiselle DA, Johnston JJ, Cheung WA, et al: Single-cell analysis of human adipose tissue identifies depot and disease specific cell types.Nat Metab 2020, 2:97-109.

24. Cavodeassi F, Modolell J, Gómez-Skarmeta JL: The Iroquois family of genes: from body building to neural patterning.Development 2001, 128:2847-2855.

25. Guo X, Liu W, Pan Y, Ni P, Ji J, Guo L, et al: Homeobox gene IRX1 is a tumor suppressor gene in gastric carcinoma. Oncogene 2010, 29:3908-3920.

26. Bennett KL, Karpenko M, Lin MT, Claus R, Arab K, Dyckhoff G, et al: Frequently methylated tumor suppressor genes in head and neck squamous cell carcinoma.Cancer Res 2008, 68:4494-4499.

27. Jiang J, Liu W, Guo X, Zhang R, Zhi Q, Ji J, et al: IRX1 influences peritoneal spreading and metastasis via inhibiting BDKRB2-dependent neovascularization on gastric cancer.Oncogene 2011, 30:44984508.

28. Lu J, Song G, Tang Q, Zou C, Han F, Zhao Z, et al: IRX1 hypomethylation promotes osteosarcoma metastasis via induction of CXCL14/NF-KB signaling.J Clin Invest 2015, 125:1839-1856.

29. Daas SI, Rizeq BR, Nasrallah GK: Adipose tissue dysfunction in cancer cachexia.J Cell Physio/ 2018, 234:13-22.

\section{Tables}

Table 1. Top 10 downregulated mRNAs and 10 upregulated mRNAs in SAT compared to VAT 
Gene id Gene name log2 Fold Change $p$ value

\section{Downregulated mRNAs}

\begin{tabular}{llll}
\hline ENSG00000184937 & WT1 & -10.42020286 & $7.24 \mathrm{E}-09$ \\
\hline ENSG00000119919 & NKX2-3 & -9.340609993 & $3.06 \mathrm{E}-12$ \\
\hline ENSG00000183242 & WT1-AS & -8.433724032 & $1.59 \mathrm{E}-10$ \\
\hline ENSG00000211892 & IGHG4 & -8.083413442 & $1.00 \mathrm{E}-09$ \\
\hline ENSG00000211668 & IGLV2-11 & -7.842925465 & $4.15 \mathrm{E}-08$ \\
\hline ENSG00000054803 & CBLN4 & -7.209658278 & $2.30 \mathrm{E}-06$ \\
\hline ENSG00000205038 & PKHD1L1 & -7.107755456 & $5.64 \mathrm{E}-06$ \\
\hline ENSG00000187848 & P2RX2 & -7.045386839 & $2.30 \mathrm{E}-06$ \\
\hline ENSG00000242221 & PSG2 & -6.846739335 & $6.57 \mathrm{E}-06$ \\
\hline ENSG00000211663 & IGLV3-19 & -6.843669107 & $9.34 \mathrm{E}-06$ \\
\hline Upregulated mRNAs & & & \\
\hline ENSG00000112246 & SIM1 & 10.22187292 & $1.84 \mathrm{E}-15$ \\
\hline ENSG00000176842 & IRX5 & 7.352859596 & $2.08 \mathrm{E}-08$ \\
\hline ENSG00000089225 & TBX5 & 6.447165153 & $2.06 \mathrm{E}-05$ \\
\hline ENSG00000170549 & IRX1 & 6.369599248 & $2.82 \mathrm{E}-22$ \\
\hline ENSG00000180318 & ALX1 & 6.250090981 & 0.000155648 \\
\hline ENSG00000180818 & HOXC10 & 6.123438559 & $7.65 \mathrm{E}-24$ \\
\hline ENSG00000251151 & HOXC-AS3 & 5.976364812 & 0.001044655 \\
\hline ENSG00000154162 & CDH12 & 5.92279196 & 0.00024887 \\
\hline ENSG00000255399 & TBX5-AS1 & 5.831484757 & $3.40 \mathrm{E}-06$ \\
\hline ENSG00000250451 & HOXC-AS1 & 5.825341418 & $3.79 \mathrm{E}-22$ \\
\hline
\end{tabular}

Table 2. ClinicalCharacteristics of 61 Participants 


\begin{tabular}{|c|c|c|c|}
\hline \multirow{2}{*}{$\begin{array}{l}\text { Clinical } \\
\text { characteristics }\end{array}$} & \multicolumn{2}{|l|}{ IRX1 } & \multirow[t]{2}{*}{$p$ Value } \\
\hline & High $(n=37)$ & Low $(n=24)$ & \\
\hline Age & $64.71 \pm 9.06$ & $65.79 \pm 8.38$ & 0.633 \\
\hline BMI & $20.36 \pm 2.12$ & $22.82 \pm 1.61$ & $0.006^{*}$ \\
\hline Weight Loss & $6.53 \pm 2.19$ & $5.12 \pm 1.93$ & $0.015^{*}$ \\
\hline Disease stage(III/IV) & $23 / 37$ & $14 / 24$ & 0.772 \\
\hline IL6(mmol/L) & $10.67 \pm 5.87$ & $7.52 \pm 4.38$ & $0.027^{*}$ \\
\hline TNFa(mmol/L) & $14.24 \pm 6.36$ & $11.80 \pm 4.11$ & $0.019^{*}$ \\
\hline Alb (g/L) & $37.89 \pm 3.43$ & $39.04 \pm 4.45$ & 0.261 \\
\hline $\mathrm{PAb}(\mathrm{mg} / \mathrm{L})$ & $207.59 \pm 52.98$ & $220.17 \pm 58.42$ & 0.093 \\
\hline $\mathrm{FAA}(\mathrm{mmol} / \mathrm{L})$ & $0.58 \pm 0.13$ & $0.53 \pm 0.17$ & 0.098 \\
\hline $\mathrm{TC}(\mathrm{mmol} / \mathrm{L})$ & $4.01 \pm 0.95$ & $4.26 \pm 1.05$ & 0.351 \\
\hline $\mathrm{TG}(\mathrm{mmol} / \mathrm{L})$ & $1.07 \pm 0.41$ & $1.10 \pm 0.37$ & 0.829 \\
\hline LDL(mmol/L) & $2.37 \pm 0.84$ & $2.60 \pm 0.91$ & 0.317 \\
\hline HDL(mmol/L) & $1.18 \pm 0.36$ & $1.25 \pm 0.57$ & 0.599 \\
\hline ApoA(g/L) & $1.16 \pm 0.28$ & $1.13 \pm 0.26$ & 0.445 \\
\hline $\mathrm{ApoB}(\mathrm{g} / \mathrm{L})$ & $0.79 \pm 0.25$ & $0.85 \pm 0.21$ & 0.268 \\
\hline ApoE(mg/L) & $35.78 \pm 14.30$ & $44.13 \pm 17.70$ & $0.047^{*}$ \\
\hline SAT area $\left(\mathrm{cm}^{2}\right)$ & $108.54 \pm 19.18$ & $127.09 \pm 24.13$ & $<0.001^{*}$ \\
\hline VAT area $\left(\mathrm{cm}^{2}\right)$ & $87.83 \pm 17.78$ & $101.09 \pm 19.85$ & $0.015^{\star}$ \\
\hline
\end{tabular}

BMI Body mass index, ALB Albumin, PAb Prealbumin, TC Totalcholesterol, TG Tri-glyceride, LDL Lowdensity lipoprotein, HDL High-density lipoprotein, ApoA Apolipoprotein A, ApoB Apolipoprotein B, ApoE Apolipoprotein E, FFA Free fatty acid, IL-6 Interleukin 6, TNF-a Tumor Necrosis Factor-a

\section{Figures}


A

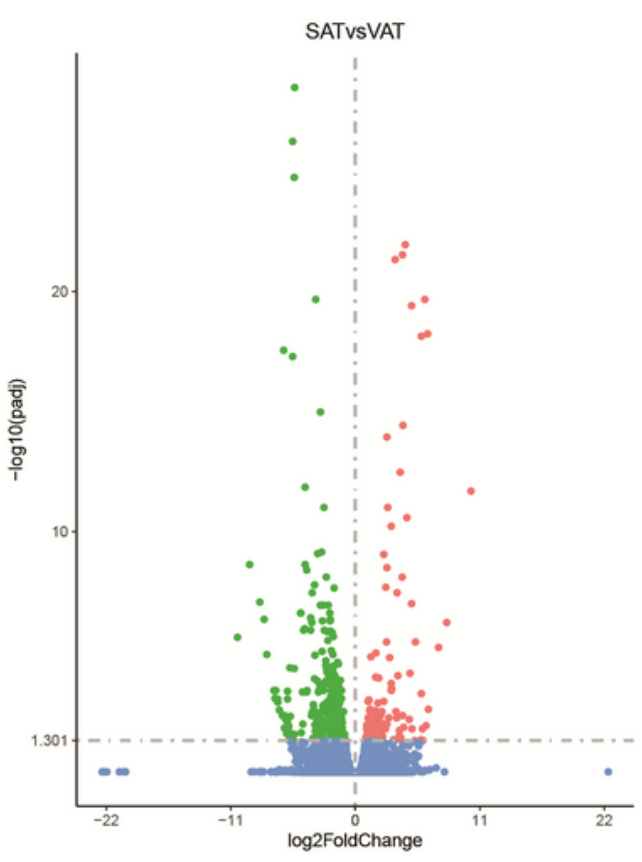

C

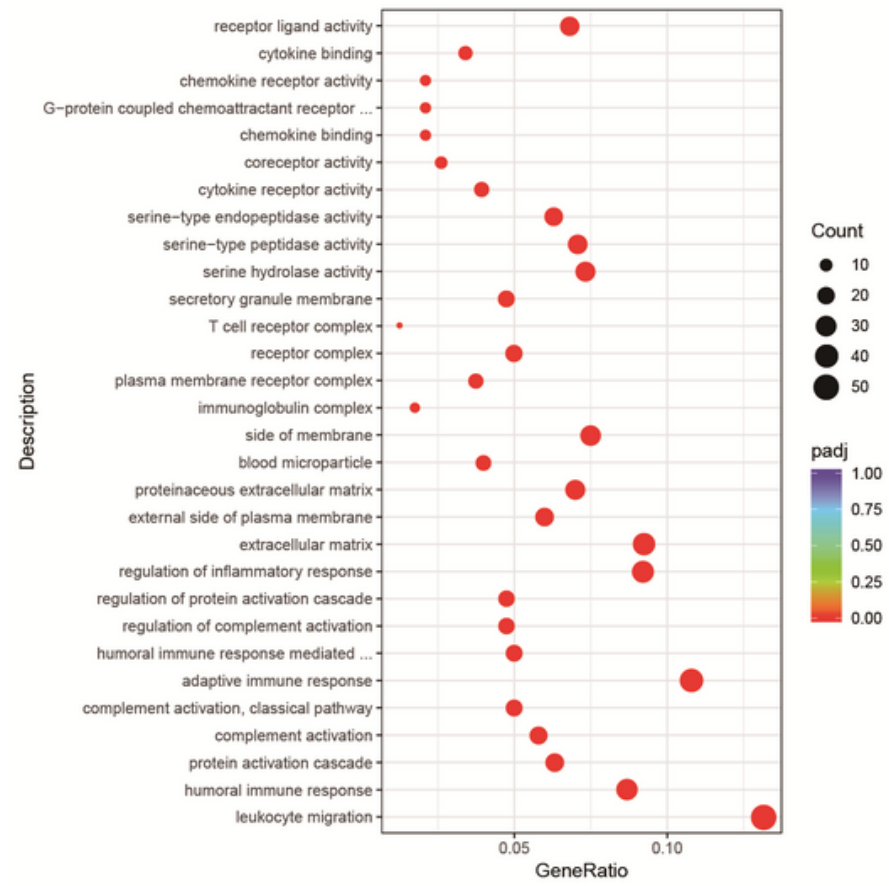

B

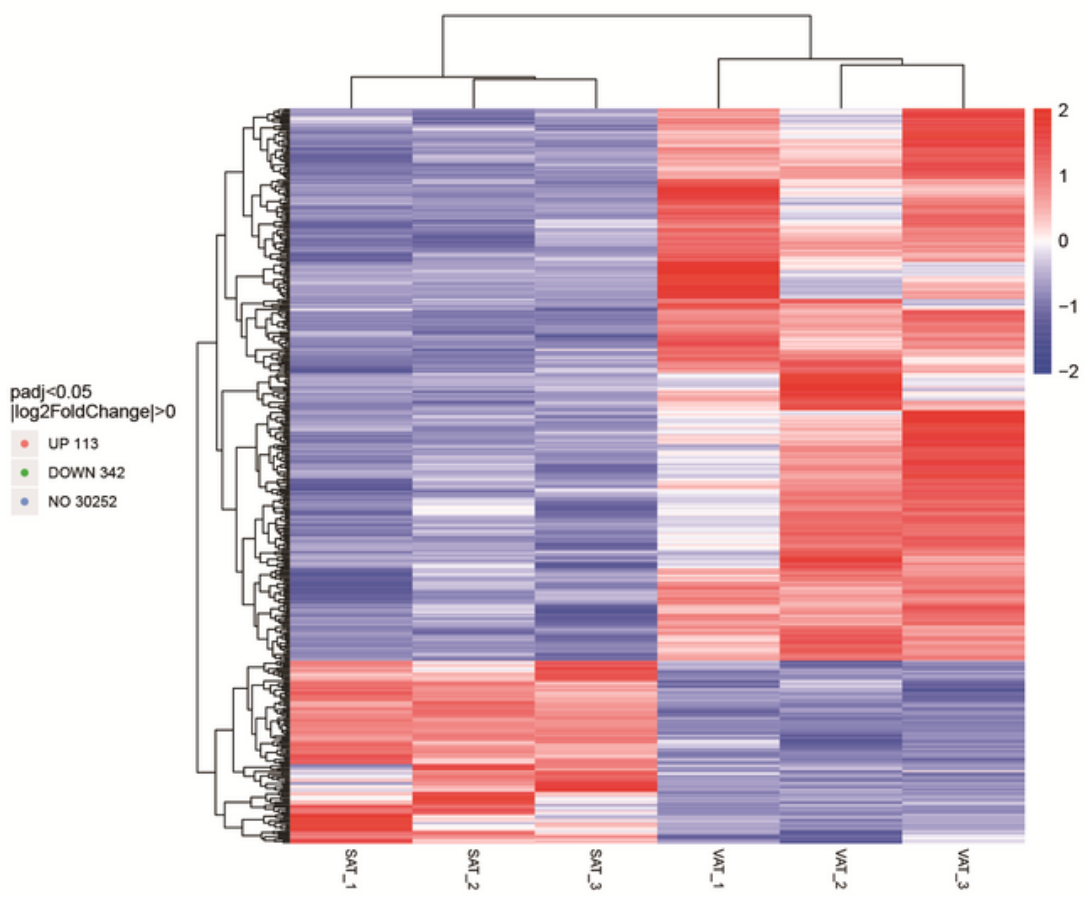

D

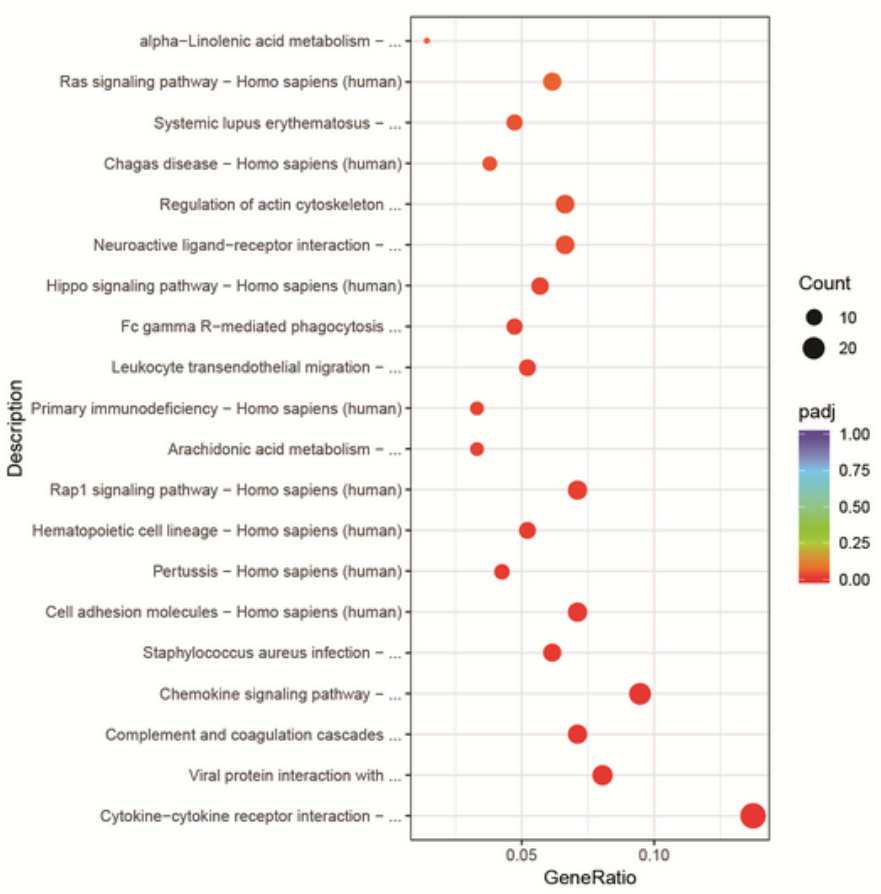

Figure 1

A total of 455 DEGs were identified as being statistically significant between SAT and VAT groups.A. Volcano plot of differently expressed genes. B. Heat map of differentiallyexpressed genes. C. Enriched GO terms. D. KEGG pathway annotations.The X-axis represented the proportion of DEGs, and the Yaxisrepresented different categories. 
A

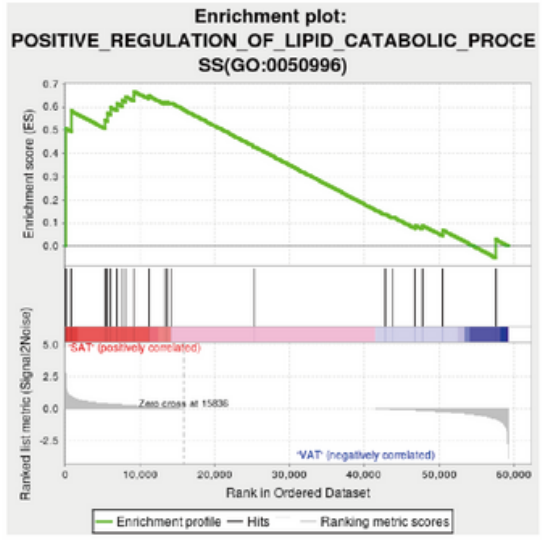

D

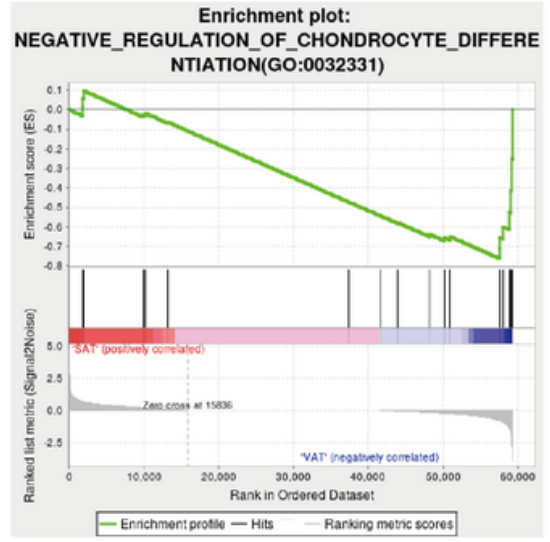

B

Enrichment plot: REGULATION_OF_FATTY_ACID_BETAOXIDATION(GO:0031998)

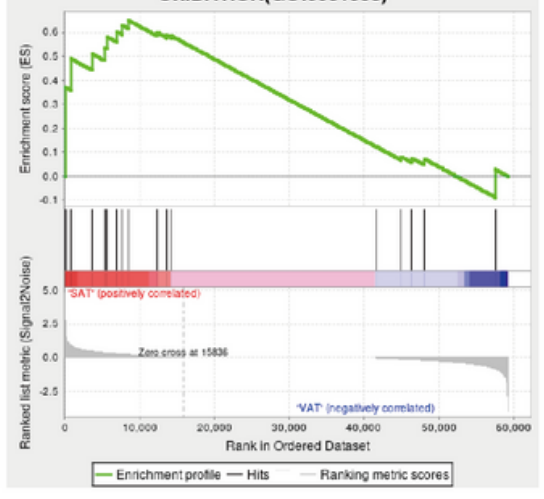

$\mathbf{E}$

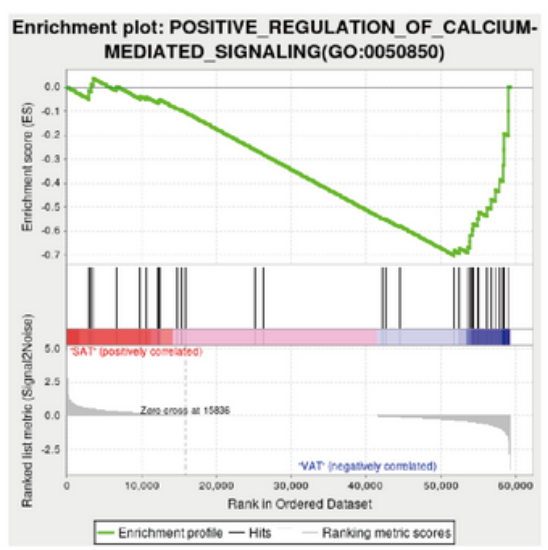

C

Enrichment plot:

POSITIVE REGULATION OF TRIGLYCERIDE METABOLI C_PROCESS(GO:0090208)

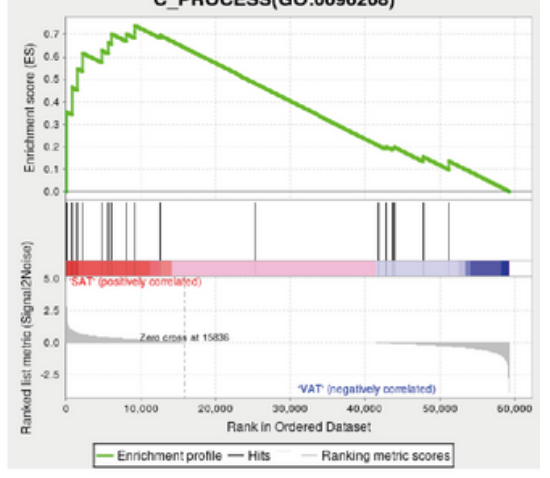

F

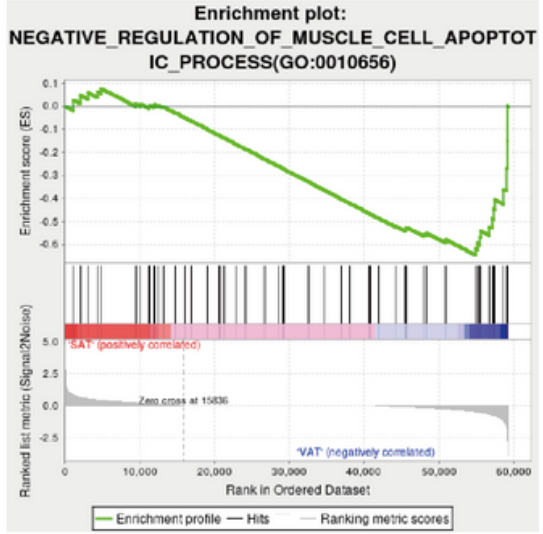

Figure 2

Enrichment plots from the gene set enrichment analysis (GSEA). (A-F) ES, enrichment score; NES, normalized ES; ADJP-val, adjusted P-value. 
A

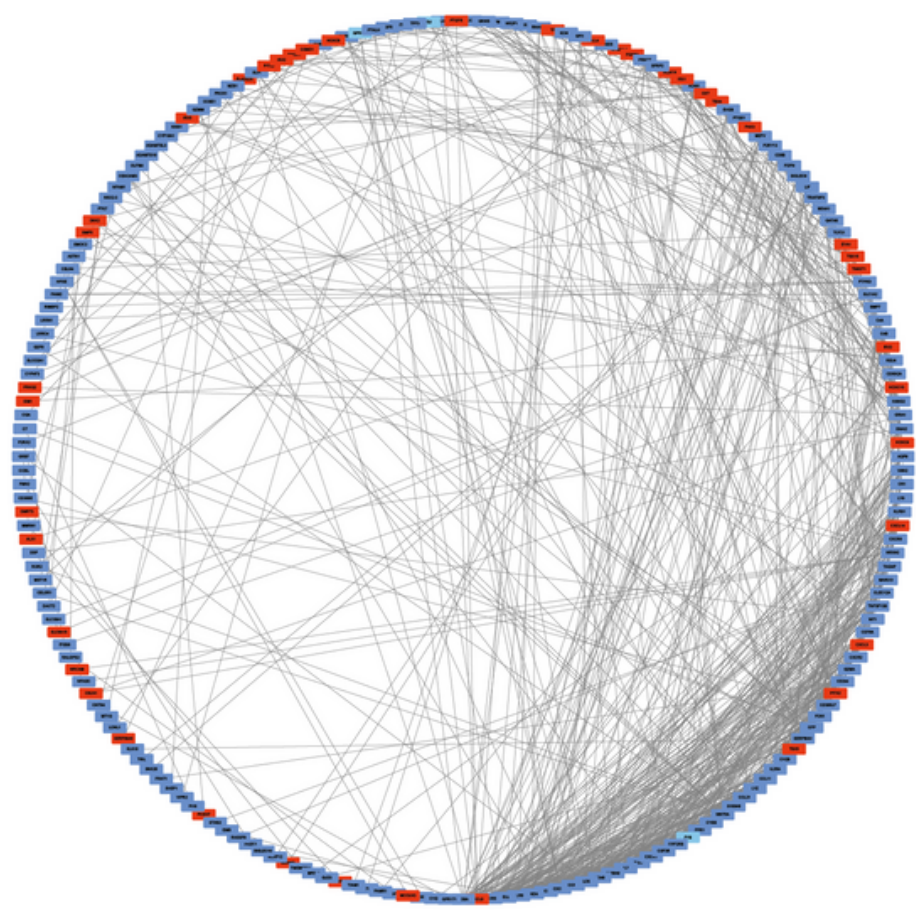

B

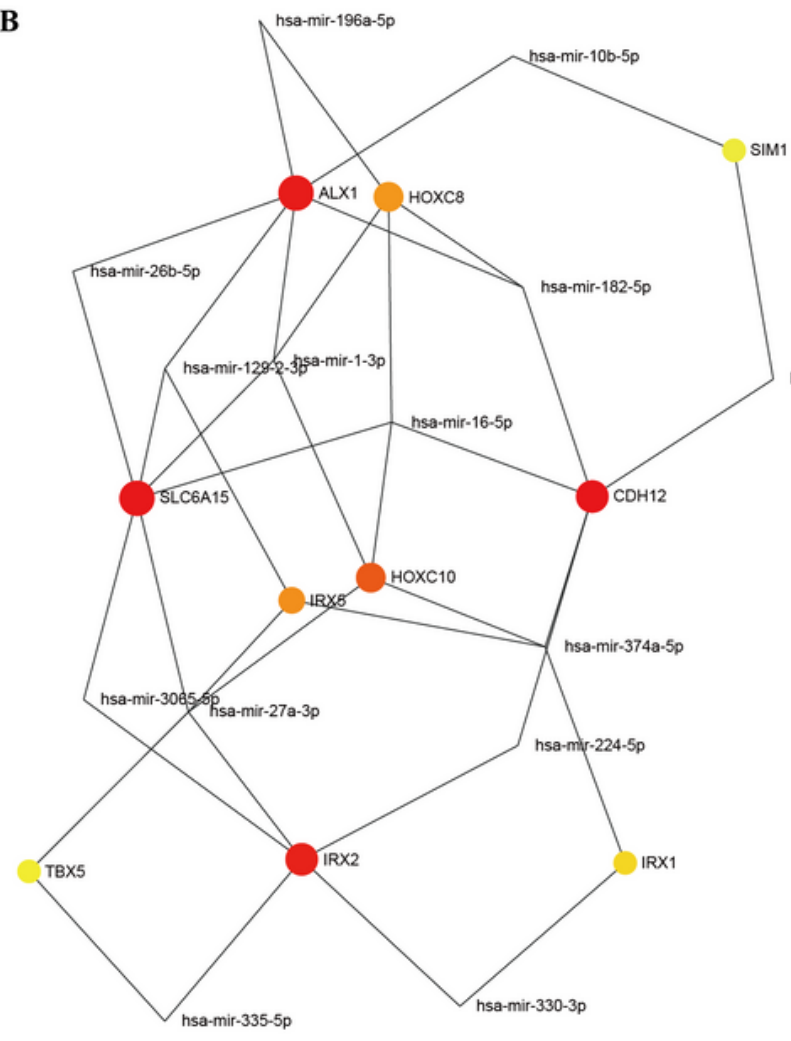

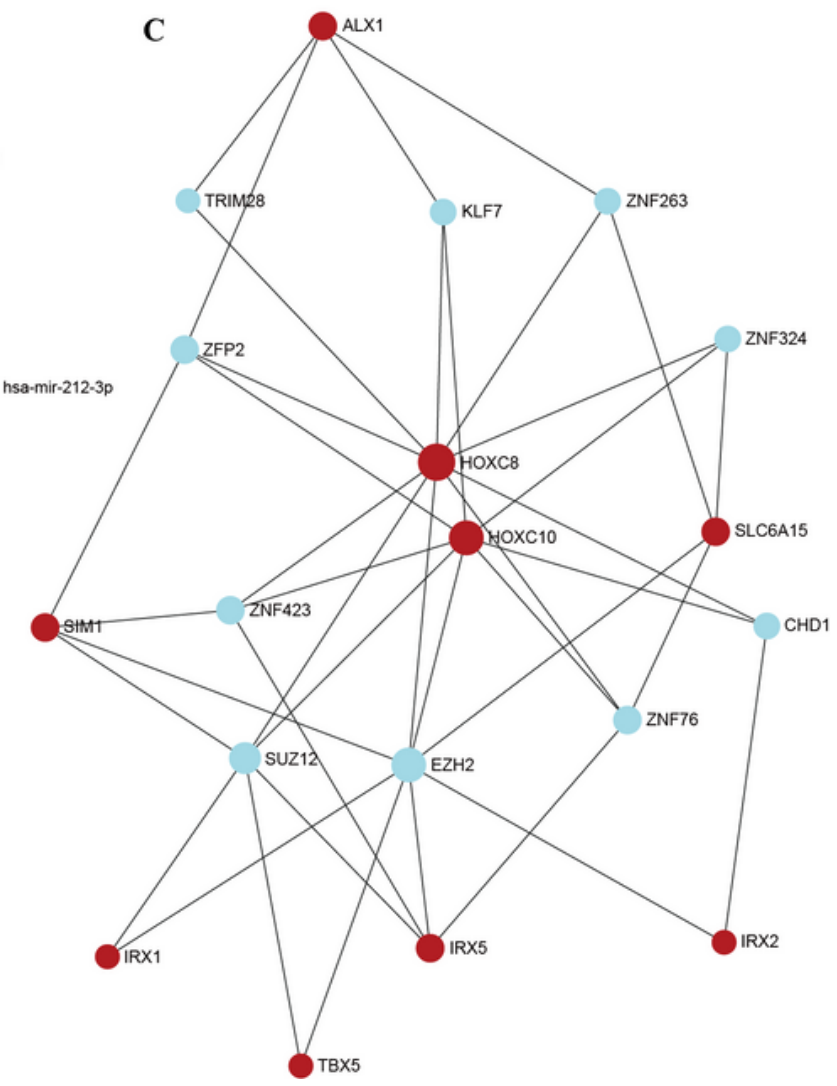

Figure 3

The PPInetwork and interaction network of DEGs between SAT and VAT. (A) The PPI network of DEGs wasconstructed using Cytoscape. (B) Interaction network linking top 10 DEGs to miRNAs. (C) Interaction network linking top $10 \mathrm{DEGs}$ to translation factors. 
A

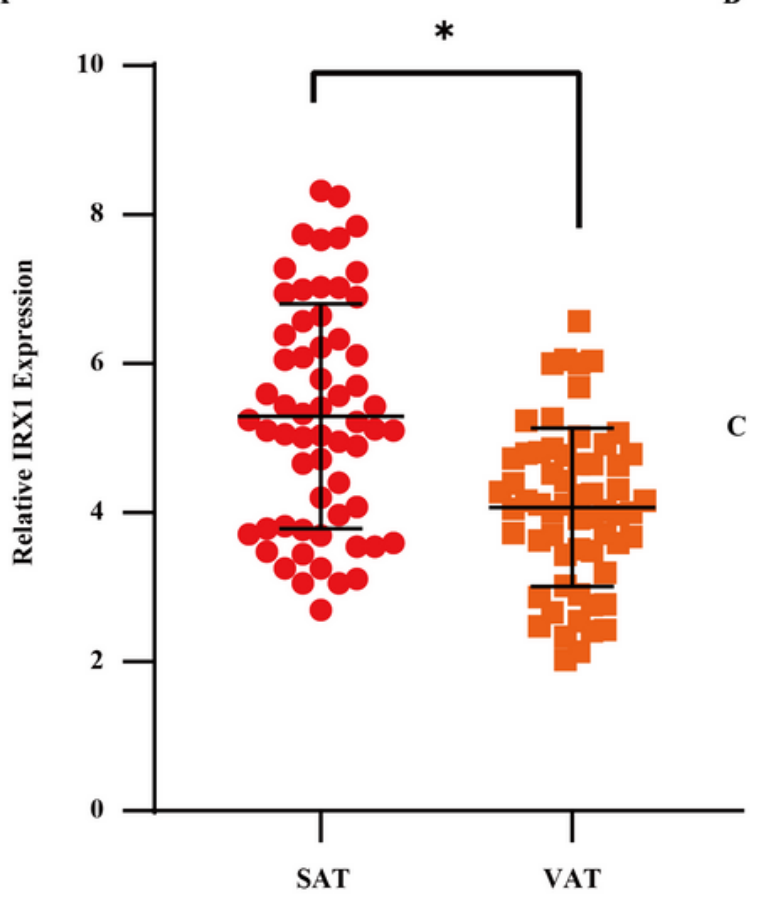

C
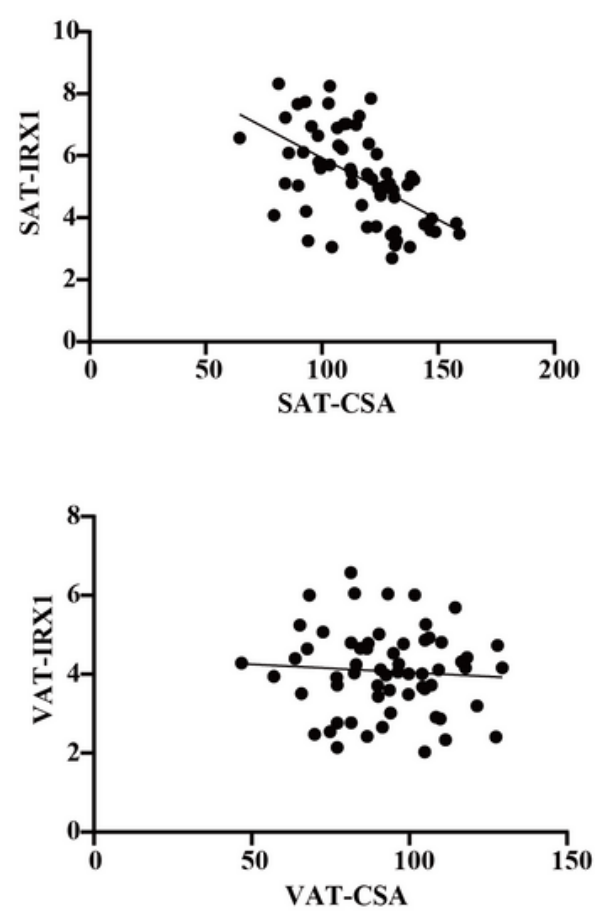

E

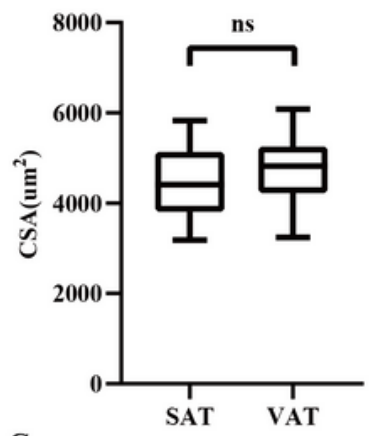

G

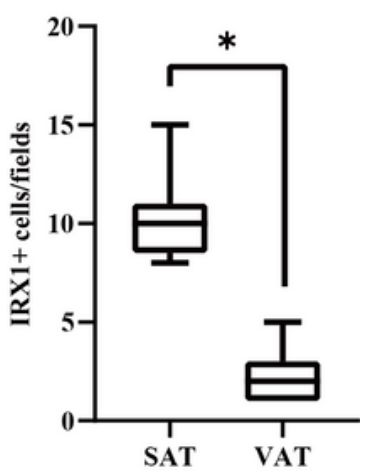

Figure 4

The expression of IRX1 was associated with pathological process in cancer cachexia. (A)Expression of IRX1 between SAT and VAT in 61 pairs of specimens. (B) Correlation between the SAT area and the expression level IRX1. (C) Correlation between the VAT area and the expression level IRX1. (D-E) Morphological evaluation of adipose tissue of SAT and VAT (hematoxylin-eosin) (Scale bar=100um). (FG) Representative images of IHCrevealing IRX1 expression in SAT and VAT (Scale bar=100um). 
A

C

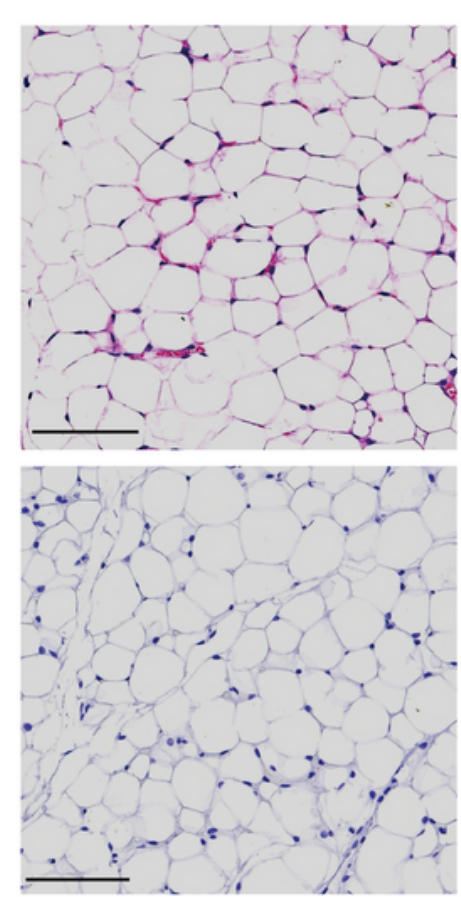

E

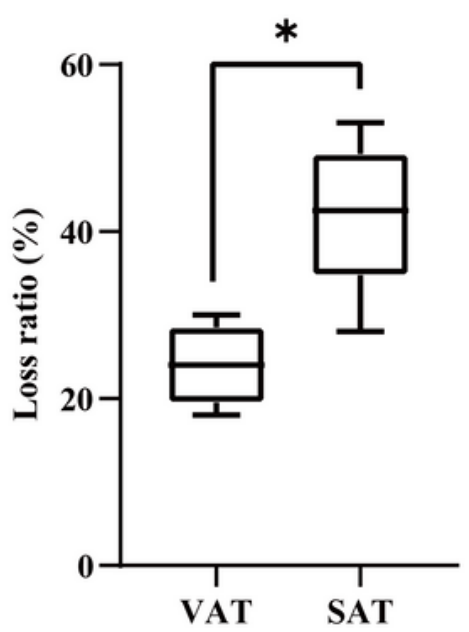

B

D
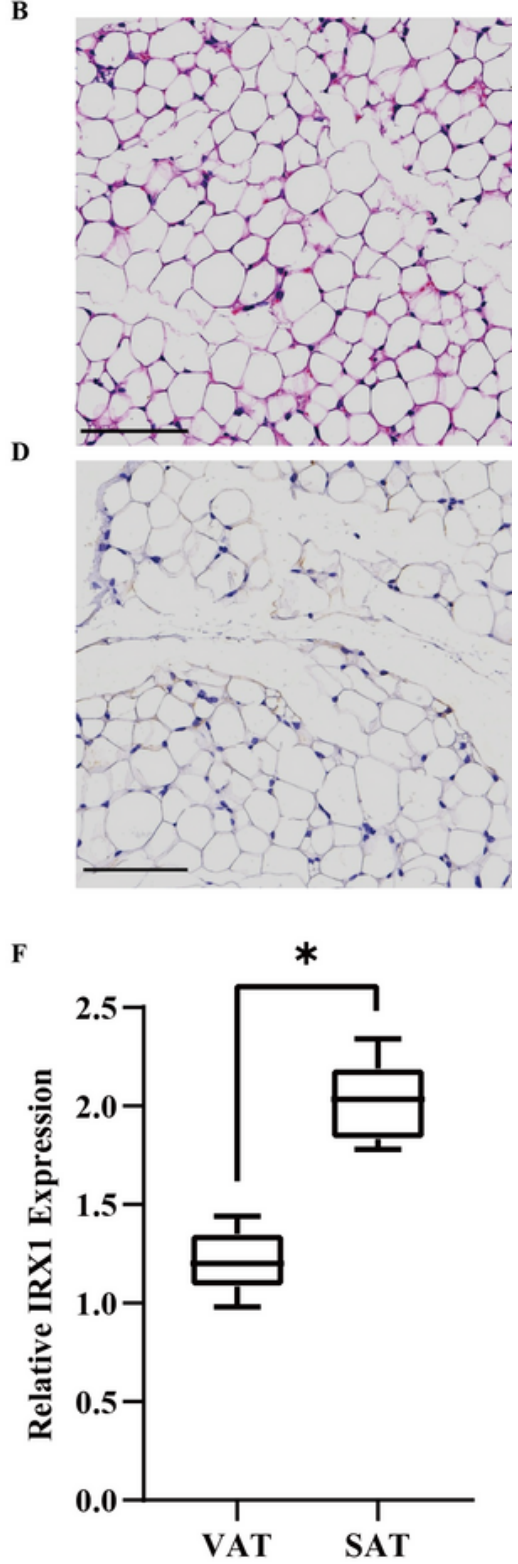

G

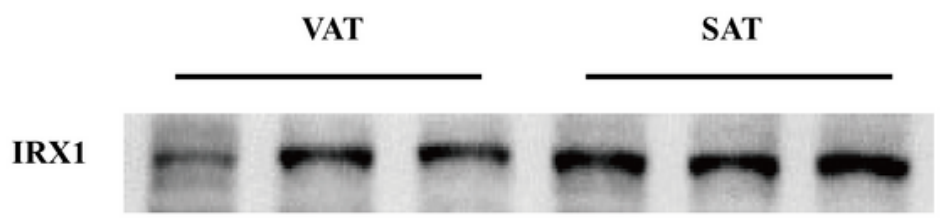

Tubulin

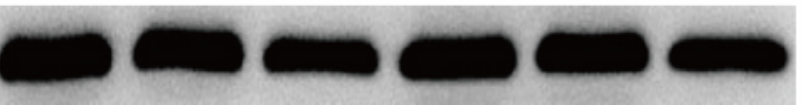

Figure 5

IRX1 was highly expressed in SAT of cachectic mice.(A-B) HE staining of SAT and VAT of mice(Scale bar=100um). (C-D) Representative images of IHC revealing IRX1 expression in SAT and VAT(Scale bar=100um). (E)Comparison of loss ratio of SAT and VAT in cachectic mice. (F)mRNA expression and (G) protein expressionlevels of IRX1 in SAT and VAT in cachecticmice. 
A

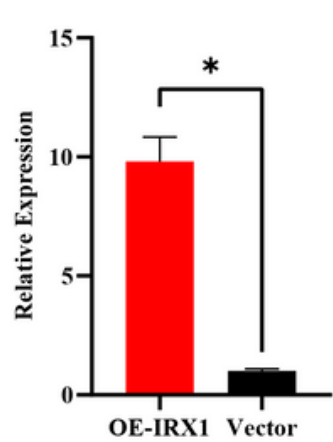

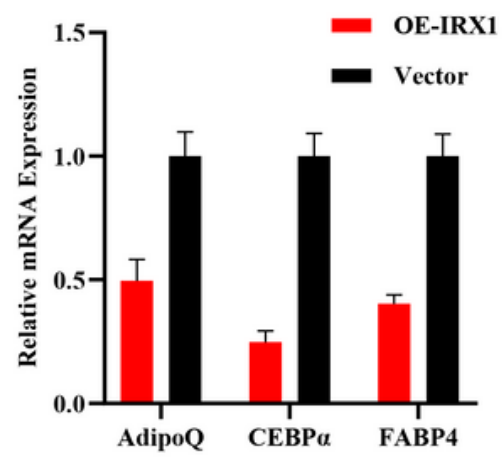

C

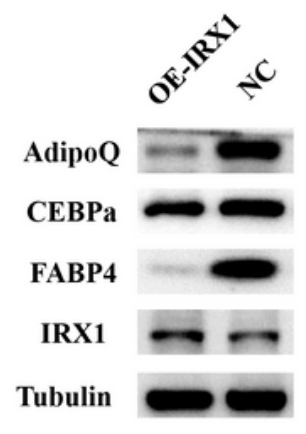

E

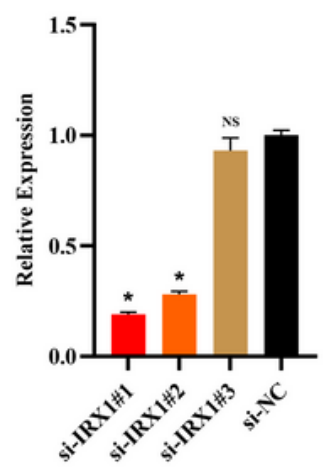

OE-IRX1

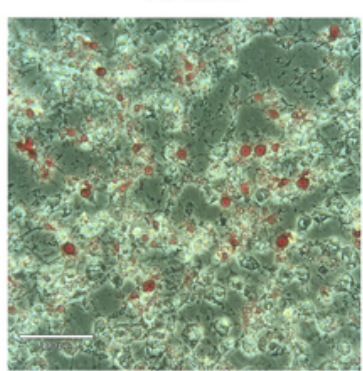

Vector

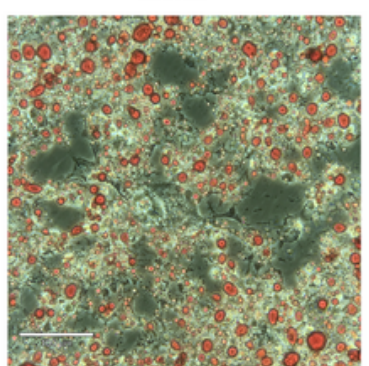

G
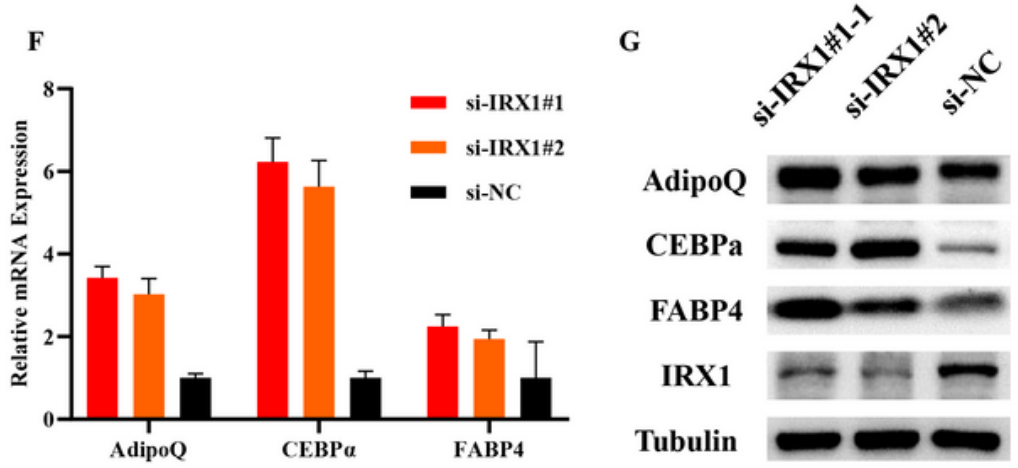

H

si-IRX1\#1

si-IRX1\#2
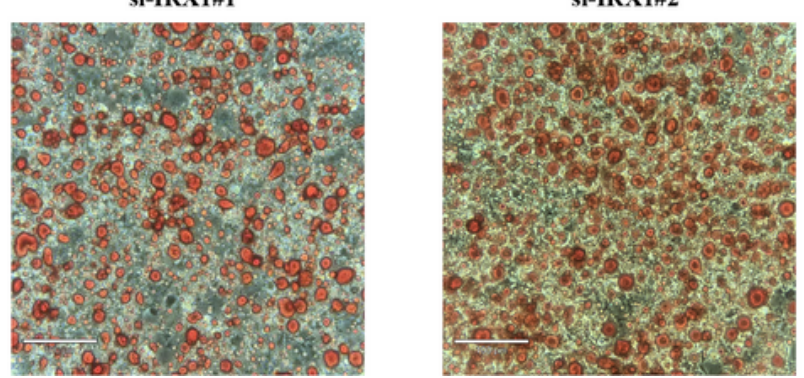

si-NC

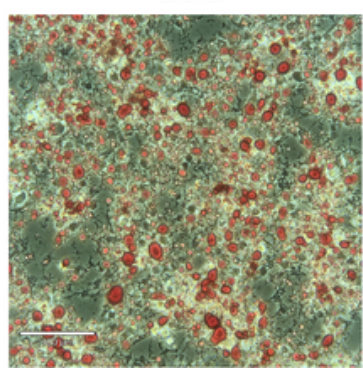

Figure 6

IRX1 suppresses adipogenesis in adipocytes. (A)Relative IRX1 expression with or without IRX1 overexpression.(B-C)qPCR and Western blot analysis of the expression of markers in adipocytes (6d) without/with overexpression of IRX1.(D) Oil Red 0 staining of lipid accumulation in adipocytes (6d)

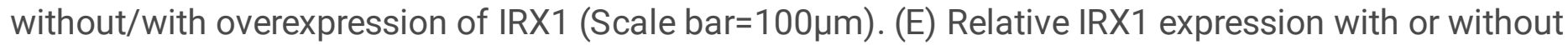
IRX1 knockdown. (F-G) qPCR and Western blot analysis of the expression of markers in adipocytes (6d) 
without/with knockdown of IRX1. (H) Oil Red $\mathrm{O}$ staining of lipid accumulation in adipocytes (6d)

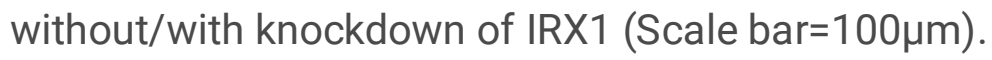

\section{Supplementary Files}

This is a list of supplementary files associated with this preprint. Click to download.

- Supplementary.docx

- Originalimage.docx 